\title{
Efecto de diferentes tratamientos térmicos sobre las propiedades tecfuncionales de la harina de fríjol blanco (Phaseolus lunatus L.) y la determinación de su potencial uso agroalimentario
} Effect of different thermal treatments on the tecfunctional
properties of white bean flour (Phaseolus lunatus L.) and
the determination of its potential agri-food use

DOI: http://doi.org/10.17981/ingecuc.15.2.2019.13

Artículo de Investigación Científica. Fecha de Recepción: 14/05/2019. Fecha de Aceptación:.25/08/2019

Yair García Pacheco

Universidad del Atlántico. Barranquilla (Colombia)

ingenieriaagroindustrial@mail.uniatlantico.edu.co

Danelys Cabrera Mercado

Universidad de la Costa. Barranquilla (Colombia) dcabrera4@cuc.edu.co

Jairo Andrés Ballestas Santos

Universidad del Atlántico. Barranquilla (Colombia)

jairoballestas10@gmail.com

Michel José Campo Arrieta

Universidad del Atlántico. Barranquilla (Colombia)

mcampo165@gmail.com

Para citar este artículo:

Y. García Pacheco, D. Cabrera Mercado, J. Ballestas Santos, M. Campo Arrieta. "Efecto de diferentes tratamientos térmicos sobre las propiedades tecfuncionales de la harina de fríjol blanco (Phaseolus Lunatus L.) y la determinación de su potencial uso agroalimentario", INGE CUC, vol. 15, no. 2, pp. 132-143, 2019. DOI: http://doi.org/10.17981/ingecuc.15.2.2019.13

\section{Resumen}

Introducción- El frijol blanco (Phaseolus Lunatus L)., es una leguminosa de alto valor nutricional, gracias a sus contenidos de proteínas, carbohidratos complejos y fibra. Es importante fomentar su consumo y diversificar su uso en el desarrollo de productos, para lo cual es necesario conocer sus propiedades funcionales y determinar su potencial uso agroalimentario.

Objetivo- Caracterizar las propiedades funcionales de la harina de frijol blanco (Phaseolus lunatus L.) sometida a diferentes tratamientos, con el fin de evaluar el efecto de los tratamientos sobre las propiedades tecnofuncionales de la harina.

Metodología- Se formuló un DCA (Diseño Completamente al Azar) donde se evaluó la composición bromatológica y funcional en harinas obtenidas a partir de frijol blanco, y los granos fueron sometidos a 3 tratamientos previos a la etapa de secado $\left(60^{\circ} \mathrm{C}\right)$, los cuales fueron: remojo $(12 \mathrm{~h})$, cocción $\left(97^{\circ} \mathrm{C}, 90 \mathrm{~min}\right)$ y escaldado $\left(72^{\circ} \mathrm{C}, 5 \mathrm{~min}\right)$.

Resultados- Los contenidos de proteína disminuyeron al aumentar el tiempo de exposición a los tratamientos. El tratamiento de cocción incrementó las propiedades de absorción de agua y aceite. El mejor resultado para la capacidad emulsificante los mostró el tratamiento de remojo con un $43,0 \%$. No se encontraron diferencias significativas entre los tratamientos de remojo y escaldado para la capacidad espumante.

Conclusiones- Las harinas sometidas a los tratamientos presentan propiedades funcionales que les confieren gran potencial para la elaboración de productos, tales como bebidas instantáneas, salsas, galletas, embutidos, productos fritos y productos de repostería.

Palabras clave- Leguminosas; proteina; secado; agroindustria; seguridad alimentaria.

\section{Abstract}

Introduction- The white bean (Phaseolus Lunatus L), is a legume of high nutritional value, thanks to its contents of protein, complex carbohydrates and fiber. It is important to promote its consumption and diversify its use in the development of products, for which it is necessary to know its functional properties and determine its potential agri-food use.

Objective- To characterize the functional properties of white bean flour (Phaseolus lunatus L.) subjected to different treatments, in order to evaluate the effect of the treatments on the techno-functional properties of the flour.

Methodology- A DCA (Completely Random Design) was formulated to evaluate the bromatological and functional composition in flours obtained from white beans, and the beans were subjected to 3 treatments prior to the drying stage $\left(60^{\circ} \mathrm{C}\right)$, which were: soaking $(12 \mathrm{~h})$, baking $\left(97^{\circ} \mathrm{C}, 90 \mathrm{~min}\right)$ and blanching $\left(72^{\circ} \mathrm{C}\right.$, 5 min).

Results- Protein contents decreased with increasing exposure time to treatments. The cooking treatment increased water and oil absorption properties. The best result for the emulsifying capacity was shown by the soaking treatment with $43.0 \%$. No significant differences were found between soaking and blanching treatments for the foaming capacity.

Conclusions- The flours subjected to the treatments have functional properties that give them great potential for the production of products such as instant drinks, sauces, biscuits, sausages, fried products and confectionery.

Keywords-Legumes; protein; drying agroindustry; food safety 


\section{INTRODUCCIÓN}

A lo largo del tiempo, la industria agroalimentaria ha venido incorporando el uso de harinas de origen vegetal como ingredientes en la preparación de productos, tales como embutidos cárnicos y productos de panificación, entre otros. Debido a su alto valor biológico los nutrientes que componen estas harinas pueden suplir los requerimientos dietarios que el producto requiere, además que su contenido proteico puede otorgarle propiedades tecfuncionales que van a determinar su uso en una formulación. Entre las harinas convencionales que más se emplean en la industria alimentaria se pueden destacar las provenientes de cereales como la de trigo, maíz, entre otros; y en el caso de las leguminosas la de soya [1].

Phaseolus lunatus L., es una leguminosa consumida como grano en África y Latinoamérica [2]. Fuente importante de proteínas, carbohidratos complejos y fibra [3], los cuales representan un sinnúmero de beneficios para la nutrición y salud humana, destacándola como una de las leguminosas de mayor consumo a nivel mundial [1]. Presenta potenciales característicos para la industria agroalimentaria, la cual continuamente busca alternativas tecnológicas y de ingredientes para la elaboración de nuevos productos alimenticios o mejorando las propiedades funcionales y organolépticas de los ya existentes, de tal modo que sean apetecibles para el consumidor y satisfagan sus necesidades nutricionales [3].

Las leguminosas utilizadas para la industria alimentaria deben ser sometidas a procesos térmicos previos a su consumo. Con los tratamientos térmicos de los compuestos antinutritivos presentes en las leguminosas son separados en su mayor proporción, mejorando propiedades como la textura y sabor de las harinas obtenidas a partir de estos granos. De igual forma, el tratamiento térmico modifica las propiedades tecfuncionales de las leguminosas, limitando su utilización en el desarrollo de formulaciones para la elaboración de productos [4].

Estudios previos han demostrado que los contenidos de antinutrientes presentes en las leguminosas, pueden ser eliminados por medio de procesos de hidratación a razón de su solubilidad en el agua. La hidratación de las leguminosas con agua destilada disminuye las concentraciones de inhibidores presentes en los frijoles (tripsina, taninos, ácido fítico y saponinas). Procesos como la cocción tienen efectos de disminución o eliminación de compuestos antinutricionales de origen proteínico e igualmente estos tratamientos mejoran la asimilación de las proteínas contenidas en las leguminosas, pero disminuye la calidad proteica del producto tratado, afectando su aprovechamiento nutricional debido a la pérdida de algunos aminoácidos esenciales [5].
El objetivo de este trabajo fue caracterizar las propiedades tecfuncionales relacionadas con la absorción de agua y grasa, la capacidad emulsionante, y la capacidad de formación de espuma y la composición nutricional de harinas a partir de los granos de Phaseolus Lunatus L., los cuales fueron sometidos a tratamientos de remojo, cocción y escaldado, previos al secado y molienda. Buscando determinar el potencial uso como materia prima en la elaboración de productos agroalimenticios. Los diferentes análisis y pruebas fueron desarrollados en el Laboratorio de Procesos Agroindustriales ubicado en el Centro de Laboratorios y Talleres de Ingeniería (CELTI) de la Facultad de Ingeniería de la Universidad del Atlántico (Colombia).

\section{REVISIÓN LITERARIA}

\section{A. Phaseolus Lunatus L.}

Es una especie muy importante para la alimentación humana que es consumida como grano. Esta leguminosa es cultivada en varios países de América y algunas regiones de Europa, Asia y África, en climas cálidos y templados, y es originaria de la América tropical y subtropical. Es conocida en diferentes partes del mundo como frijol lima, comba, pallar, haba pallar o frijol mantequilla; en Colombia es denominado también como frijol lima o zaragoza. Phaseolus lunatus $L$. es una leguminosa con un alto contenido nutricional entre los que resaltan los carbohidratos, proteínas, fibra, hierro y calcio [6].

\section{B. Influencia de los tratamientos térmicos en los alimentos}

Los tratamientos térmicos son técnicas que ayudan a su conservación, disminuyendo la carga microbiana, transformando su estructura y las propiedades físicoquímicas de los mismos, otorgándoles una mejor digestibilidad. Además, pueden derivar efectos variables sobre los hidratos de carbono en cuanto a los almidones que se gelatinizan, y también influyen en la desnaturalización de las proteínas. Por otra parte, estos tratamientos pueden afectar la composición nutricional y las propiedades funcionales del producto final. La desnaturalización parcial o total de ciertas proteínas se puede producir debido al calor que se aplica a los alimentos, aumentando la digestibilidad de estas, aunque también conlleva a una disminución de la calidad nutricional [7].

Las leguminosas al ser sometidas a tratamiento con calor se deben controlar estrictamente el tiempo y la temperatura, para no afectar la estructura, de manera que si el tratamiento térmico aplicado es inadecuado se produce la desnaturalización total de la proteína generando su inactividad e insolubilización [8]. 


\section{Propiedades tecfuncionales}

Son propiedades que proporcionan información acerca del comportamiento de los ingredientes dentro de un producto alimenticio, en los que se encuentran las proteínas, carbohidratos, fibras, entre otros; de manera que cada una de estas propiedades ayudan a determinar su campo de aplicación en la industria agroalimentaria [9].

Dentro de las propiedades tecfuncionales es posible identificar las que tienen relación con la rehidratación y contenido de agua, tales como la capacidad de absorción de agua y la capacidad de retención de agua; con el contenido de lípido se relaciona la capacidad de retención de aceite. También se presentan la capacidad de hinchamiento, gelificación, espumante y emulsionante, que son otras de las propiedades funcionales que permiten predecir su uso como ingrediente en la industria agroalimenticia. Estas propiedades se ven afectadas al someterse a diferentes procesos térmicos, debido a que se generan modificaciones físico-químicas, alteraciones en la estructura y desnaturalización de las proteínas, afectando propiedades tales como las capacidades de retención de agua y aceite, formación de espuma, de emulsiones y gelificación [3].

La capacidad de absorción de agua es la cantidad de agua retenida al material hidratado bajo la acción de una fuerza externa. Esta propiedad le confiere una mejor textura en los productos cárnicos como las salchichas, por lo que le transmite mayor consistencia, viscosidad y propiedades adhesivas. La capacidad de absorción de agua depende de la concentración y estructura de la proteína del ingrediente, que a su vez estas son afectadas al ser sometidas a tratamientos térmicos. De igual manera, esta propiedad puede influir de gran importancia en la elaboración de productos de panificación como las galletas porque les confiere una suave textura [3].

La capacidad de absorción del aceite de la proteína es muy importante para la elaboración de productos, debido a que estos polímeros mejoran la retención de sabor y el gusto, además que es importante para la conservación de aroma y el aumento de los tiempos de la vida útil de alimentos de panadería, repostería y bollería, tal como a alimentos cárnicos y productos instantáneos. Esta propiedad es estrechamente significativa en la fabricación de productos fritos a razón del poder oxidativo y en el de enranciamiento [3].

La capacidad de formación de espumas es la facultad que poseen las proteínas de crear capas rodeadas de una interface agua-aire donde se incorpora mediante fuerza de agitación mecánica debido a la no disminución de los componentes de la espuma [10]. Las proteínas con muy alta capacidad de formación de espumas son muy utilizadas para la elaboración de productos tradicionales tales como panificación, repostería y en la preparación de postres. La capacidad de formación de espuma depende de la solubilidad de la proteína lo cual esta puede ser afectada al ser sometida a tratamientos térmicos, por la desnaturalización de las proteínas, generando así una disminución de la espuma [9].

La capacidad emulsificante expresa la suficiencia que poseen las proteínas contenidas en un producto para generar una dispersión compuesta por una fase grasa en un medio acuoso. La funcionalidad de los productos alimenticios está determinada en gran medida por las características particulares de la emulsión que pueden generar las proteínas. No obstante, la formación de emulsiones es influenciada por compuestos no proteicos como los carbohidratos y las fracciones de fibra, que pueden favorecer sustancialmente esta propiedad, las harinas cuyas proteínas presenten buena capacidad de formar emulsiones podrán ser empleadas en la elaboración de productos en las líneas de salsamentarías, embutidos y aderezos [3].

\section{Efectos del tratamiento térmico de harina de leguminosa en las propiedades tecfuncionales}

Los tratamientos térmicos afectan en mayor o menor medida las propiedades funcionales de los alimentos, ocasionando cambios en su estructura. La fracción de almidón es afectada en mayor proporción por el procesamiento térmico, ocasionando su gelatinización, modificando la estructura y tamaño de los granos, afectando como consecuente la viscosidad de los productos que se elaboren aparatar de estas harinas. Estos cambios dan lugar a variaciones en algunas propiedades tales como la absorción de agua, capacidad de hinchamiento y gelificante [11].

Las proteínas también son afectadas por los procesamientos térmicos, al ocasionar cambios en sus moléculas. La desnaturalización por causa de los tratamientos térmicos provoca un desenrollamiento y rompimiento en las estructuras secundaria, terciarias y cuaternarias de las proteínas, quedando simplemente la estructura primaria, las cuales se terminarán perdiendo por agregación y precipitación, generando como consecuente efectos de alteraciones en propiedades funcionales como la capacidad de retención de agua, aceite, espumante y emulsificante [12].

\section{Metodología}

\section{A. Materia prima}

Se utilizaron granos de Phaseolus Lunatus L. variedad blanco, sanos sin manchas negras o perforaciones por insectos, los cuales fueron obtenidos de un cultivo experimental de $200 \mathrm{~m}^{2}$ establecidos en la granja Pitorro ubicada en el corregimiento de Campeche-Baranoa 
(Latitud: 10.732369, Longitud:-74.915444), con características agroecológicas de suelos franco-arenosos, con una temperatura promedio de $33^{\circ} \mathrm{C}$, y una altura sobre el nivel del mar de 118 metros y precipitaciones entre 450 y $1200 \mathrm{~mm}$ promedio año. Se tomaron tres muestras aleatorias en el cultivo y los granos fueron pesados y empacados en 3 muestras iguales de 500 $\mathrm{g}$ para su posterior procesamiento en el Laboratorio de Procesos Agroindustriales ubicado en el Centro de Laboratorios y Talleres de Ingeniería (CELTI) de la Facultad de Ingeniería de la Universidad del Atlántico.

\section{B. Procesamiento del grano}

Los granos fueron sometidos a 3 tratamientos (remojo, cocción y escaldado) con el fin de evaluar su efecto sobre la composición bromatológica y propiedades tecfuncionales de la harina. Se formuló un diseño completamente al azar de los tres tipos de pretratamientos que fueron sometidos los granos. Todos los ensayos se realizaron por triplicado.

Remojo: Los granos se colocaron en $600 \mathrm{ml}$ de agua destilada durante 12 horas. Luego, fueron secados en un deshidratador WESTON de referencia 75-0201-W a temperatura de $60^{\circ} \mathrm{C}$, hasta llegar a un peso constante con la curva de secado del modelo de Page [13]. Una vez fueron secados los granos, estos fueron llevados a un molino eléctrico BRENTWOOD de referencia CG152, hasta obtener un polvo fino. El polvo obtenido de la molienda fue pasado por un tamiz para obtener un tamaño de partícula de $150 \mu \mathrm{m}$.

Cocción: Los granos fueron sometidos a cocción a temperatura de $98^{\circ} \mathrm{C}$ por un tiempo de 1 hora y 30 minutos. Luego, las etapas de secado, molienda y tamizado, se realizaron en forma similar a la descrita anteriormente.

Escaldado: Los granos fueron sometidos a procesamiento térmico a una temperatura de $72^{\circ} \mathrm{C}$ durante 5 minutos. Y después fueron secados, molidos y tamizados en forma similar a los procesos anteriores.

Finalmente, las harinas obtenidas fueron empacadas en bolsas de polietileno con cierre hermético, aisladas de la luz con papel aluminio y almacenadas a temperatura ambiente en un lugar seco para evitar oxidaciones hasta el momento de sus análisis.

\section{Determinación de las propiedades bromatológicas de la harina}

A continuación, se presenta la metodología que fue utilizada para la determinación de las propiedades bromatológicas de las 3 muestras.

Humedad: Se determinó por diferencia de peso antes y después del secado de la muestra hasta que alcanzó peso constante [14].

Proteína: El contenido se determinó utilizando el método Kjeldahl [15].
Cenizas: El contenido de ceniza se determinó por mineralización de las muestras en una mufla [14].

Grasas: Se empleó el método de extracción Soxhlet [14].

Fibra: El contenido de fibra se determinó [14].

Carbohidratos: El contenido de carbohidratos totales fue determinado por diferencia [16].

\section{Caracterización de las propiedades tecnofuncionales de la harina}

Una vez obtenidas las harinas se determinaron las propiedades funcionales de las 3 muestras para conocer la influencia de los tratamientos sobre las mismas.

Capacidad de Absorción de Agua (CAA): Se mezcló $1 \mathrm{~g}$ de muestra con 10,0 $\mathrm{ml}$ de agua destilada y se dejó en reposo durante 30 minutos a temperatura ambiente. Luego en una centrifuga HETTICH de referencia ROTOFIX 32A se colocaron en centrifugación a $3000 \mathrm{rpm}$ durante 30 minutos. Posteriormente, el sobrante fue desechado y la diferencia entre la masa inicial de la muestra y la masa final después de pasar por la centrifuga, fue la cantidad de agua absorbida. Los resultados se expresaron en g de agua retenidos/g de muestra [17].

Capacidad de Absorción de Aceite (CAA): Se mezclaron 10,0 $\mathrm{ml}$ de aceite de palma con 1,0 g de muestra. Luego se dejó en reposo durante 30 minutos a temperatura ambiente. Posteriormente, se centrifugó a 3000 rpm durante 30 minutos. Por último, el sobrenadante se desechó y la diferencia entre la masa inicial de la muestra y la masa final después de pasar por la centrifuga, fue la cantidad de aceite absorbido. Los resultados fueron expresados en $\mathrm{g}$ de aceites retenidos/g de muestra [17].

Capacidad de Formación de Espumas (CES): Se colocaron en suspensión 2,0 g de muestra en 100,0 ml de agua destilada. Luego fueron batidas en una licuadora por 2 minutos. Se registraron los volúmenes antes (V1) y después (V2) del batido. La capacidad espumante (CES) se calculó de acuerdo con la ecuación (1).

$$
C E S=(\%)=\frac{(\mathrm{V} 1-\mathrm{V} 2)}{\mathrm{V} 1} \times 100
$$

Capacidad emulsificante: Se preparó una dispersión con 1,4 g de muestra y 20,0 ml de agua destilada. De esta se tomó 5,0 $\mathrm{mL}$ para luego ser homogenizada con 5,0 $\mathrm{ml}$ de aceite de palma durante 1 minuto. Posteriormente, las muestras fueron centrifugadas a 2000 rpm por 10 minutos [18]. Se midió la altura en $\mathrm{cm}$ de la capa emulsificadora (ACE) y del contenido total del tubo (ACT). La capacidad emulsificante (CEM) se calculó mediante la ecuación (2) [17].

$$
\operatorname{CEM}(\%)=\frac{\mathrm{ACE}}{\mathrm{VCT}} \times 100
$$




\section{E. Análisis estadísticos}

Las curvas de secado de los granos de P.lunatus, se modelo utilizó el software Python 3.5.1 (Python Software Foundation).

Los resultados obtenidos de los ensayos se tabularon y analizaron usando el programa estadístico Stargrafics ${ }^{\circledR}$ Centurión XVI. Mediante un análisis de varianza, ANOVA, y con la prueba de Tukey se determinó como influyen los niveles en las variables de respuesta.

\section{F. Determinación de uso potencial de las harinas}

Se categorizaron 6 grupos de productos alimenticios: Bebidas instantáneas, productos fritos, embutidos, productos de repostería, salsamentaría y galletería; los cuales utilizan harinas como ingredientes para su elaboración. A través de una revisión bibliográfica, se pudo identificar las propiedades funcionales esenciales que deben poseer las harinas para cada uno de estos grupos y los valores en los cuales deben encontrarse las mismas.

Luego, se construyó una tabla donde se situaron las 4 propiedades que fueron evaluadas y los respectivos rangos de valores en los cuales deberían encontrarse las harinas tratadas (establecidos según la bibliografía hallada) para cada uno de los grupos de productos.

Por último, teniendo en cuenta los resultados obtenidos en la caracterización de las propiedades tecfuncionales, se ubicaron las harinas tratadas que hayan presentado los mejores resultados y sus valores se encuentren dentro de los establecidos, para así ser sugeridas como potencial uso en la elaboración de dichos productos.

\section{Resultados}

\section{A. Determinación bromatológica de la harina}

La Fig. 1 muestra el ajuste de los datos experimentales de los granos de P. Lunatus a los modelos del secado Page, con un $\mathrm{R}^{2}$ de 0,99896 , alcanzando la humedad en equilibrio de $7,9 \%, 7,1 \%$ y $6,6 \%$, a tiempos de 11,16 y 14 horas con una temperatura de $60^{\circ} \mathrm{C}$, para los tratamientos de remojo, escaldado y cocción respectivamente.

Se puede observar que los tiempos para alcanzar la humedad en equilibrio muestran una fuerte influencia de los pretratamientos, mostrando un menor tiempo para el remojo de los granos de P. Lunatus. Se puede notar que la perdida de humedad fue más rápida al inicio del secado y pasadas las 6 horas la perdida de humedad es muy variable para los tres tratamientos.

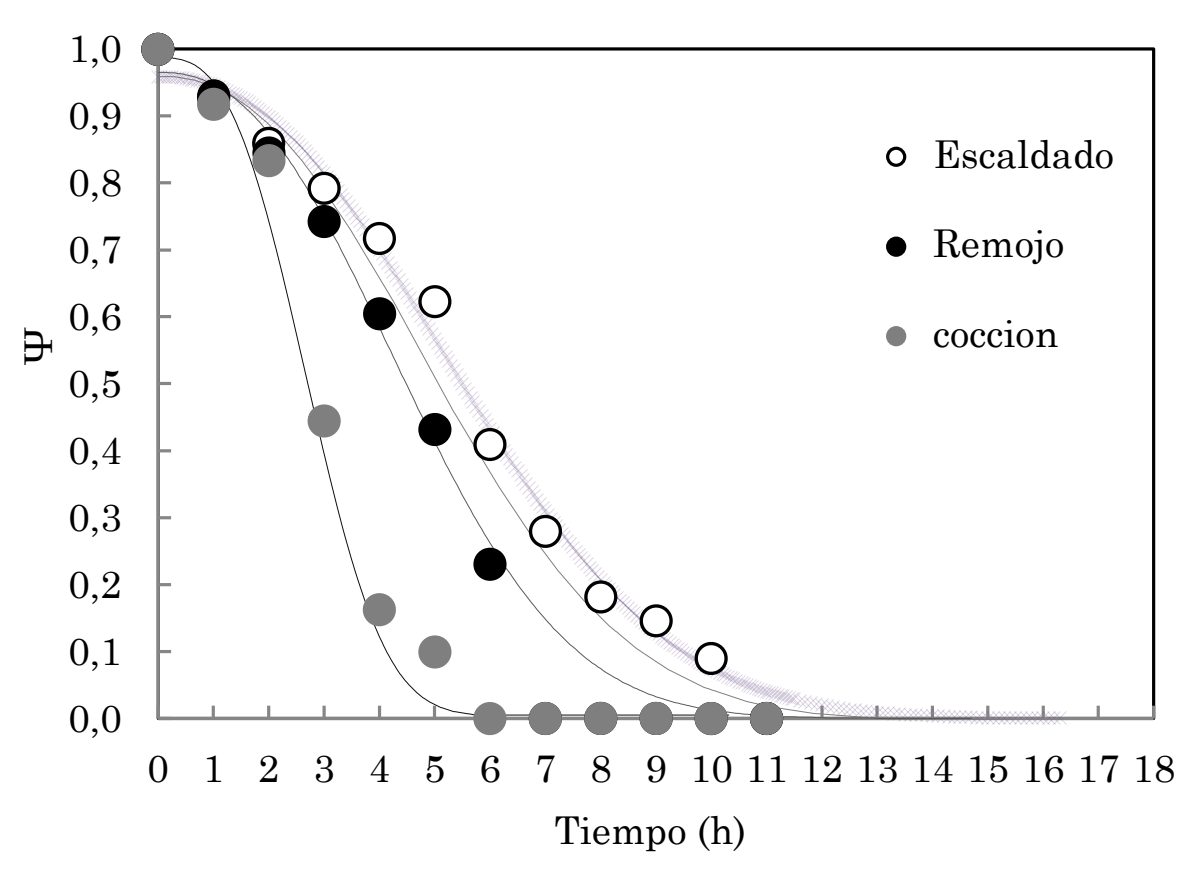

Fig. 1. Curvas de secado del $P$. Lunatus. para los tratamientos a temperatura de $60^{\circ} \mathrm{C}$. Fuente: Autores.

Tabla 1. Caracterización BROMatológiCa de las harinas DE $P$. LUNATUS PARA CADA UNO DE LOS TRATAMIENTOS.

\begin{tabular}{|c|c|c|c|}
\hline \multicolumn{5}{c}{ Tratamientos } \\
\hline Análisis & Remojo & Escaldado & Cocción \\
\hline Humedad (\%) & $7,9 \pm 0,27 \mathrm{a}$ & $7,1 \pm 0,30$ & $6,6 \pm 0,18$ \\
\hline Cenizas (\%) & $4,4 \pm 0,13$ & $4,5 \pm 0,17$ & $2,9 \pm 0,46 \mathrm{a}$ \\
\hline Grasa (\%) & $0,9 \pm 0,07$ & $1,3 \pm 0,01 \mathrm{a}$ & $1,1 \pm 0,07$ \\
\hline Proteína (\%) & $18,3 \pm 0,05 \mathrm{a}$ & $17,3 \pm 0,09$ & $16,5 \pm 0,03$ \\
\hline Fibra (\%) & $4,0 \pm 0,03$ & $4,3 \pm 0,12$ & $4,2 \pm 0,02$ \\
\hline Carbohidratos (\%) & $64,5 \pm 0,50$ & $65,6 \pm 0,08$ & $68,7 \pm 0,60 \mathrm{a}$ \\
\hline
\end{tabular}

Nota: Medias con letras en una misma fila muestran diferencias significativas $(p \leq 0,05)$.

Fuente: Autores.

\section{B. Determinación bromatológica de las harinas}

En la Tabla 1, se presentan los resultados correspondientes a la determinación bromatológica realizada a las harinas de frijol blanco (P. Lunatus), sometidas a tratamientos de remojo, escaldado y cocción.

Humedad. Se puede observar en la Tabla 1, que el contenido de humedad de las harinas de P. Lunatus sometida a procesamiento de remojo, escaldado y cocción, fue de 7,9, 7,1 y 6,6\% respectivamente. Apreciando una reducción en el contenido de humedad en la harina sometida al tratamiento de cocción. Como en otros estudios [28], la harina elaborada a partir de judía pinta presentó una humedad de 7,8\% cuando fue colocada en remojo y disminuyó hasta 7,5\% cuando fue sometida a procesamiento de remojo-cocción [3]. La disminución del contenido de humedad se debe a factores tales como tiempo y temperatura de secado o a los procesamientos a los que fueron sometidos los granos. Los procesamientos generan cambios en la 
estructura, textura y capacidad de retención de agua, causada por desnaturalización de las proteínas y gelatinización de los almidones [18]. Estos tratamientos también pueden generar pérdida de humedad por procesos de deshidratación osmótica [19].

Cenizas. Los valores de ceniza oscilan entre 2,9 y $4,4 \%$. Estos resultados son muy próximos a otros reportes para harina de diferentes variedades de leguminosa [28] con valores entre 2,7 y 5,2\% [3], los cuales reportaron para variedades de $P$. Vulgaris valores entre 2,6 y 3,9\% con respecto al tratamiento de cocción [4]. El menor contenido de ceniza, se encontró para la harina sometida a tratamiento cocción con un valor de $2,9 \%$, presentando una destacada reducción. Este comportamiento que experimenta la harina se debe a la pérdida de minerales por lixiviación que ocurre en el proceso de cocción [20].

Grasas. Los procesamientos de remojo, escaldado y cocción influyen sobre el contenido de grasa ocasionando su disminución. Los porcentajes de grasa obtenidos en el presente estudio oscilan entre 0,9 y 1,3\%. Estos valores fueron inferiores a reportes anteriores [4] de 2,2\% y 6,2\%, para variedades de Phaseolus Vulgaris, crudas y procesadas.

Se han reportado valores desde $1,4 \%$ hasta $3,2 \%$, para harinas de granos crudos y fermentados-cocidos de diferentes variedades de frijol [21] [11], mientras otros valores muy cercanos oscilan entre $1,0 \%$ y $1,1 \%$, en harina de granos de quinchoncho (Cajanus cajan (L.) Millsp.), sometidos a diferentes procesamientos [2].

Proteína. El contenido de proteína disminuyó al aumentar el tiempo de exposición al tratamiento, debido a la solubilización de nutrientes [4], por lo que en promedio el contenido de proteína arrojado fue de 17 , $4 \%$. Este valor se encuentra ente los rangos de 15,1\% y $25,9 \%$ para harinas de diferentes variedades de leguminosa crudas y procesadas [3].

Por otro lado, se ha determinado que los distintos procesamientos disminuyeron significativamente el contenido de minerales, reportando valores que oscilan entre $23,1 \%$ y $31,3 \%$, para cuatro variedades de harina de Phaseolus Vulgarus crudas y procesadas [4]. Se reportaron procesamientos de los granos que no modificaron significativamente el contenido de proteína, produciendo valores de $16,9 \%$ a $19,9 \%$ en harina de granos de quinchoncho (Cajanus cajan $(L$.) Millsp.) [2]. Existen reportes con valores entre 22,0 y $26,7 \%$ [27], para harinas de granos crudos, cocidos, fermentados y fermentados-cocidos de diferentes variedades de frijol [21], siendo estos valores superiores a los obtenidos en el presente estudio.

Este comportamiento está determinado por factores tales como la variedad, especie, cultivo, composición nutricional [3], desnaturalización parcial o total de las proteínas al someterse los granos a procesamiento, el cual afecta la composición nutricional y las propiedades funcionales del producto final [7].

El procesamiento con calor causa la desnaturalización de las proteínas, afectando propiedades las cuales posen una relación con la composición fisicoquímica de los productos tratados. La desnaturalización ocasiona la solubilización de nutrientes, causando que las diferentes propiedades funcionales se vean alteradas, como las capacidades espumantes y emulsificantes [12].

Fibra. La harina obtenida de los granos sometidos a tratamiento de remojo presentó menor contenido de fibra, con respecto a los tratamientos de escaldado y cocción. Estos resultados indican que los granos sometidos a proceso de escaldado y cocción influyen sobre el contenido de fibra de la harina.

Este comportamiento coincide con resultados donde se reportaba que el contenido de fibra aumentaba con la cocción debido a la presencia de almidón resistente retrogradado para variedades de Phaseolus Vulgarus [4]. El contenido de fibra aumentó con el tiempo de fermentación de un 8,3\% hasta 9,5\% [22], mientras las etapas de remojo y cocción influyen sobre el contenido de fibra de la harina, causando un incremento promedio de 25,0\% [2].

Los tratamientos térmicos pueden ocasionar diversos efectos en el contenido de fibra tales como cambios en la composición, aumento en el contenido de fibra y solubilización de las fibras, como resultado de la generación de ciertos compuestos como almidones resistentes y de la ruptura de sus componentes, favoreciendo la interacción de estos con sustancias como proteínas y lípidos [23].

Carbohidratos. El Contenido de carbohidratos se estimó por diferencia a partir de los valores obtenidos del resto de componentes químicos, de manera que su comportamiento será resultado de la variación de dichos componentes. Se aprecian valores de 64,7 y $68,7 \%$ respectivamente, estos se encuentran entre los rangos de 70,0 y 81,0\% reportados [28], para variedades de leguminosas [3] y a su vez estos son superiores a los reportados para harina de granos de quinchoncho (Cajanus cajan (L.) Millsp.), sometidos a diferentes procesamientos [2].

Este comportamiento es consecuencia del menor contenido de proteína presente en la muestra de $P$. Lunatus y de las diferentes fracciones que componen los carbohidratos tales como el contenido de almidón, fibras y carbohidratos solubles, por lo que estas fracciones son fundamentales en la determinación de las propiedades tecfuncionales [24]. 
Propiedades tecfuncionales como la capacidad de absorción de agua dependen del comportamiento que tiene la fracción de almidón al someterse a tratamiento térmico [25]. Este va a provocar modificaciones en su estructura cristalina y gelatinización parcial o total, ocasionando un aumento de su volumen debido a la vibración de las moléculas del almidón, el cual causa ruptura de los puentes de hidrógenos provocando hinchamiento por la absorción de agua y la viscosidad debido a la formación de un gel por la adhesión de unos gránulos a otros [26].

\section{Caracterización de las propiedades tecfuncionales de la harina}

En la Tabla 2, se encuentran consignados los datos obtenidos en cada de una de las pruebas funcionales realizadas para los 3 tratamientos a los que fueron sometidas la harina de frijol blanco.

Capacidad de absorción de agua (CAA). En la Tabla 2 se puede apreciar que existen diferencias significativas en el tratamiento de cocción con respecto a los otros 2 tratamientos, arrojando un valor de 3,3 $\mathrm{g} / \mathrm{g}$ para este, mientras que el escaldado y el remojo lograron valores similares entre sí de 2,3 y 2,4 g/g respectivamente.

La capacidad de absorción de agua se incrementó, producto de la cocción de los granos previa al secado. Estos valores se asemejan a los obtenidos para el garbanzo Sinaloa [28], el cual obtuvo como resultado una CAA de 2,2 g/g para el grano sometido a remojo y de $3,3 \mathrm{~g} / \mathrm{g}$ para el de remojo-cocción [3].

Estudios previos han demostrado que el tratamiento térmico desnaturaliza las proteínas incrementando la accesibilidad a la misma y en consecuencia a sus aminoácidos polares, los cuales tienen una gran afinidad por el agua, produciéndose un incremento en la capacidad para absorber agua [3].

Además de las proteínas, los carbohidratos por su naturaleza hidrofílica, la gelatinización del almidón y el hinchamiento de la fibra dietética también son contribuyentes a este incremento en la capacidad para absorber agua al ser sometidas a procesos térmicos [21].

Los resultados obtenidos por parte del grano sometido a cocción con valores más altos de CAA en comparación con los otros 2 tratamientos se encuentran relacionados directamente con los obtenidos en la caracterización bromatológica.

El contenido de la proteína para el grano en cocción fue de $16,5 \%$, donde este fue el de menor valor entre los 3 tratamientos analizados. Esto quiere decir, que hubo una mayor desnaturalización de la proteína y esto a su vez facilitó una mayor cantidad de agua absorbida.
La gelatinización del almidón contenido en los granos por efecto del calentamiento térmico también provocó el comienzo de un proceso lento de absorción de agua en las zonas intermicelares amorfas que son menos organizadas y a la vez las más accesibles. Además, el alto contenido de fibra que arrojó este tratamiento $(4,2 \%)$ en relación con los otros, potenció la capacidad de absorber agua.

Estos valores de CAA fueron más bajos que los encontrados para el frijol Phaseolus Vulgaris de variedad DOR-500 crudo, con 2,8g/g y cocido 4,4 g/g [27].

Capacidad de Absorción de Aceite (CAA). Como se puede observar en la Tabla 2, existen diferencias significativas en el tratamiento de cocción con respecto a los otros 2 tratamientos, alcanzando un valor de 2,6 $\mathrm{g} / \mathrm{g}$, mientras que por su parte el escaldado y el remojo tuvieron valores similares entre sí de 2,1 y $2,0 \mathrm{~g} / \mathrm{g}$ respectivamente.

Las variaciones de la CAG dependen del comportamiento de las interacciones hidrófobas de las proteínas como resultado del tratamiento térmico y la capacidad del almidón y la fibra para adsorber el aceite [28]. La diferencia de estos valores de CAA para los diferentes tratamientos, se debe a la desnaturalización de las proteínas ocasionada por la cocción de los granos que provocó alteraciones en su estructura favoreciendo la retención física de la grasa, lo cual parece ser producto de un entrampamiento físico de las grasas por parte de las proteínas, a través de la formación de estructuras denominadas micelas y que generan una mayor CAG para este tratamiento [21]. Además, el contenido de carbohidratos totales y fibras en mayor proporción con respecto a los otros 2 tratamientos pudo haber influido también en dichas variaciones.

Los resultados obtenidos de esta investigación coinciden con otros reportados [2], a partir de una caracterización de las propiedades funcionales del grano de quinchoncho (Cajanus cajan (L.) Millsp.) sometiéndolo a condiciones similares y que al igual que el presente trabajo los valores de CAG para granos que fueron procesados con cocción antes del secado $(2,0 \mathrm{~g} / \mathrm{g})$ aumentaron con respecto a los granos que no tuvieron tratamientos térmicos previos $(1,8 \mathrm{~g} / \mathrm{g})$.

Tabla 2. Propiedades funcionales de las harinas de $P$. LUNATUS SOMETIDA A DIFERENTES TRATAMIENTOS.

\begin{tabular}{|c|c|c|c|c|}
\hline & \multicolumn{3}{|c|}{ Propiedades Funcionales } & \\
\hline Tratamiento & CAA (g/g) & CAG (g/g) & CES (\%) & CEM (\%) \\
\hline Remojo & 2,4 & 2,0 & 14,0 & $43,8 \mathrm{a}$ \\
\hline Cocción & $3,3 \mathrm{a}$ & $2,6 \mathrm{a}$ & 3,2 & 6,5 \\
\hline Escaldado & 2,3 & 2,1 & 14,7 & 20,9 \\
\hline
\end{tabular}

Nota: Medias con letras en una misma columna muestran diferencias significativas $(p \leq 0,05)$.

Fuente: Autores. 
Esta propiedad es muy importante para la formulación de productos de panadería, productos cárnicos y sustitutos de carne, sopas y alimentos para freír ya que se relaciona con la capacidad de retención de los sabores y con la suavidad que adquiere el producto [2].

Capacidad Emulsificante (CEM). La capacidad emulsificante siempre suele ser afectada por efecto de tratamientos térmicos, debido a la desnaturalización de las proteínas. Sin embargo, una desnaturalización parcial, suele mejorar las propiedades emulgentes, debido al incremento de la flexibilidad molecular y de la hidrofobia superficial [17].

En la Tabla 2, se puede apreciar que existen diferencias significativas en el tratamiento de remojo en relación con los otros 2 tratamientos. Los granos que fueron colocados en remojo y que por ende no sufrieron tratamiento térmico previo, arrojaron un porcentaje de CEM del 43,8\%. En cambio, por su parte los granos que fueron sometidos a escaldado y cocción presentaron porcentajes de CEM mucho más bajos de 21,0 y $6,5 \%$ respectivamente.

Estos valores elevados de CEM encontrados para el grano en remojo se relacionan principalmente con las proteínas. El mayor contenido de proteína encontrado entre los 3 tratamientos fue en el de remojo (18,3\%), debido a que no sufrió de desnaturalización proteica por efecto de algún tratamiento térmico previo a la etapa de secado, a diferencia de los otros dos procesamientos (cocción y escaldado), dando como resultado una mayor capacidad emulsificante por parte de este.

Estos resultados se asemejan a otros [28], que para la lenteja pardina, obtuvieron un porcentaje de CEM de $47,0 \%$ para el grano crudo y un $5,0 \%$ para el sometido a procesamiento de remojo-cocción. Este comportamiento lo relacionan con el contenido de fibra alimentaria y la proporción en la que las fracciones de fibra se encuentran presentes en cada especie de leguminosa [3].

Por otro lado [27], se determinaron la capacidad emulsificante para los granos de frijol Vigna sinensis de variedades Orituco y Tuy (crudos y tratados), encontrándose con que los granos que fueron sometidos a cocción no presentaron dicha capacidad, pero que al ser tratados en combinación con un proceso de fermentación natural, produjo que el grano conservara su capacidad de emulsificar, respaldando dicho comportamiento bajo la hipótesis de que el proceso de fermentación pudo haber impedido la total desnaturalización de las proteínas al momento de que los granos pasaran por el proceso de cocción [21].
"La habilidad de las proteínas para contribuir con la formación y estabilización de la emulsión es particularmente importante en la elaboración de productos emulsionados como mayonesas, salsas y de productos cárnicos, donde actúan como cohesores de las fases lipídicas y acuosas" [2].

Capacidad Espumante (CES). Como se puede observar en la Tabla 2, no se muestran diferencias significativas para la capacidad espumante entre los tratamientos remojo y escaldado (14,0 y $14,7 \%$ respectivamente). En cambio, el tratamiento de cocción si presentó diferencias significativas con respecto a los otros 2 tratamientos arrojando el porcentaje de CES más bajo con tan solo $3,2 \%$. Al aplicar procesos térmicos ocurre desnaturalización proteica, que, junto con la agitación o el batido a velocidades altas, puede disminuir el poder espumante del alimento proteico, debido a la agregación y precipitación de las proteínas [3]. Los bajos valores de CES que presentó el grano sometido a cocción corresponden en mayor proporción a las proteínas. Las modificaciones en la disposición de las moléculas de proteínas al someterse a altas temperaturas, conllevaron a que la harina procesada con cocción tuviera un bajo poder espumante [3].

Para 2 variedades de la especie Tigernut (Cyperus esculentus) sometida a procesos de germinación se encontraron valores en la capacidad de espuma que van desde $10,2 \%$ hasta $12,9 \%$ [29].

Por otra parte, se determinaron las capacidades espumantes para el frijol Phaseolus Vulgaris de variedad papa, obteniendo un porcentaje de $30,5 \%$ para el grano crudo y 11,0\% para el sometido a cocción. Estos valores se deben al contenido de proteína hallado, que a su vez también fueron superiores a los encontrados en esta investigación. Para el grano crudo fue de $26,5 \%$ y para el cocido de $25,4 \%$ [4].

Por su parte [28], también se obtuvo valores más altos en la capacidad espumante para el garbanzo Sinaloa y la lenteja Pardina. Los granos que fueron puestos en remojo arrojaron un porcentaje de 25,0 y $40,0 \%$ y los granos en cocción 8,0 y $20,0 \%$, respectivamente [3].

\section{Determinación de uso potencial de las harinas}

En la Tabla 3, se presentan las propiedades funcionales (CAA, CAG, CEM y CES) y los respectivos rangos para cada uno de los grupos de productos (bebidas instantáneas, repostería, embutidos, salsas, galletería y productos fritos), en los que deberían encontrarse los valores de las harinas para ser empleadas en la elaboración de dichos productos. La capacidad para absorber agua juega un papel muy importante para la elaboración de bebidas instantáneas y galletas, pues 
les va a conferir una mejor textura en el caso de las galletas. El valor de referencia de la CAA para harinas comerciales es de 2,1 a $3,7 \mathrm{~g} / \mathrm{g}$ [30].

Las harinas con valores de absorción de agua mayores a 3,0g de agua/g de muestra, pueden favorecer la textura de productos de panificación como las galletas, tortas y panes, representando una alternativa importante para enriquecer mezclas de harinas en la elaboración de estos productos [31].

Por otro lado [13], elaboraron una bebida láctea instantánea a base de harina de arracacha, en donde el valor promedio de CAA para esta bebida instantánea fue de $3,1 \mathrm{~g} / \mathrm{g}$, el cual tuvo una aceptación en cuanto a sus características fisicoquímicas, funcionales y sensoriales [32], después de evaluar una mezcla de harinas de plátano y guandul que presentó una CAA de $3,5 \mathrm{~g} / \mathrm{g}$, que consideran que estas harinas pueden ser recomendadas como potencial ingrediente para la elaboración de una bebida instantánea. La capacidad de absorción de aceite es una propiedad que se hace deseable especialmente en productos para freír como donuts, empanadas; donde ayuda a favorecer la retención del sabor, además disminuye el desarrollo de la rancidez oxidativa y en consecuencia aumenta la estabilidad durante el almacenamiento. La mayoría de los granos exhiben una capacidad de absorción de aceite inferior a 5,0 g de aceite/g con un alto margen de variación [33].

En 2011, se encontró que para la harina de Vitabosa (Mucuna deeringiana) sin ser sometida a ningún tratamiento, el valor de CAG fue de $2,1 \mathrm{~g} / \mathrm{g}$ [5]. También se sugiere como ingrediente para la formulación de productos fritos a la harina de guandul (Cajanus cajan) sometida a procesamientos de remojo-cocción cuya CAG fue de 2,0 g/g [2] [33]. Para los grupos de salsas y embutidos, que la harina utilizada para la elaboración de estos productos tenga una buena capacidad emulsificante es fundamental. Las harinas estudiadas [28] tuvieron una CEM entre 23,0 y 47,0\% y que además afirma que estas pueden tener un potencial uso alimenticio [3].

Para la harina de frijol Phaseolus Vulgaris de diversas variedades sometidos a proceso de fermentación- cocción obtuvieron valores de CEM entre 35,5 y $58,3 \%$ y también aseguran que estas harinas pueden ser usadas en productos que requieran una buena capacidad emulsificante [4].

En los productos de repostería, la capacidad de formación de espuma es esencial para su elaboración. Para las dos variedades de la especie Tigernut ( $C y$ perus esculentus) sometida procesos de germinación, encontraron valores en la capacidad de espuma que van desde $10,2 \%$ hasta $12,9 \%$, sugiriéndolas para como potencial uso en la elaboración de productos de repostería y confitería [29]. Otra recomendación para la elaboración de productos de repostería es determinar los porcentajes de CES de 16,9\% y 27,0\% para las variedades de Phaseolus Vulgaris, perdicita y vaina morada, respectivamente [4].

Una vez ubicadas las harinas tratadas que presentaron los mejores resultados y cuyos valores se encuentran dentro de los rangos establecidos en la tabla 3 , se construyó la Tabla 4, con el fin de determinar el potencial uso de las harinas obtenidas con el tratamiento (Cocción, remojo y escaldado) aplicado a los granos de P. Lunatus.

TABla 3. CATEgORIZACIÓN DE LAS PROPIEDADES FUNCIONALES DE LAS HARINAS SEGÚN PRODUCTOS A ELABORAR.

\begin{tabular}{|c|c|c|c|c|c|c|}
\hline & \multicolumn{5}{|c|}{ Productos } \\
\hline Propiedades Funcionales & Bebidas inst. & Repostería & Embutidos & Salsas & Galletería & Productos Fritos \\
\hline CAA & $3,0-3,5 \mathrm{~g} / \mathrm{g}$ & - & - & - & $3,0-3,5 \mathrm{~g} / \mathrm{g}$ & - \\
\hline CAG & - & - & - & - & - & $2,0-3,0 \mathrm{~g} / \mathrm{g}$ \\
\hline CEM & - & - & $35,0-59,0 \%$ & $35,0-59,0 \%$ & - & - \\
\hline CES & - & $10,0-40,0 \%$ & - & - & - & - \\
\hline
\end{tabular}

Fuente: [3], [4], [29], [30], [31], [32], [33].

TABla. 4. ClasifiCACiÓN DE LAS HARINAS OBTENIDAS PARA LA ELABORACIÓN DE PRODUCTOS.

\begin{tabular}{|c|c|}
\hline Producto & Harinas Tratadas \\
\hline Bebida inst. & Cocción \\
\hline Repostería & Escaldado ó remojo \\
\hline Embutidos & Remojo \\
\hline Salsamentaría & Remojo \\
\hline Galletería & Cocción \\
\hline Productos fritos & Cocción \\
\hline
\end{tabular}

Fuente: Autores. 


\section{CONCLUSIONES}

El procesamiento térmico provoca una disminución en la composición nutricional de la harina de frijol $P$. Lunatus, en el caso de la proteína en la harina sometida a cocción que tuvo un porcentaje de $16,5 \%$ en comparación con el remojo $18,3 \%$ que no fue sometida a tratamiento térmico. Las propiedades tecfuncionales se ven afectadas por causa de la desnaturalización de las proteínas. La absorción de agua incrementa debido a la gelatinización de la fracción de almidón, al ser sometido a tratamiento térmico. El contenido y tipo de fibra también tiene incidencia en las propiedades emulsificantes y de absorción de agua.

El tratamiento de cocción incrementa las propiedades de absorción de agua y aceite en un 30\% y $23 \%$ respectivamente en relación con los otros dos tratamientos, pero disminuye notablemente las emulsificantes y espumantes. Los granos en remojo al no ser sometidos a calentamiento presentaron valores de emulsión con un $43,8 \%$, sin embargo, no presentó los mejores resultados en cuanto a absorción de agua y aceite. El procesamiento de escaldado solo mostró los mejores resultados para la propiedad espumante con $14,7 \%$, aunque no presentó diferencias significativas con el tratamiento de remojo para esta propiedad.

Las harinas de frijol blanco Phaseolus Lunatus L. sometidas a procesos de remojo, cocción y escaldado presentan propiedades funcionales que les confieren gran potencial para ser usadas en la elaboración de productos alimenticios.

Las harinas tratadas con cocción pueden ser utilizadas para la elaboración de productos tales como bebidas instantáneas, galletas y productos fritos. Las harinas colocadas en remojo podrían incorporarse en productos como salsas y embutidos; y las sometidas a escaldado pueden ser utilizadas para la elaboración de productos de repostería.

\section{REFERENCIAS}

[1] J. Umaña, C. Alvarez, S. Lopera y C. Gallard, "Caracterizción de harinas alternativas de origen vegetal con potencial aplicación en la formulación de alimentos libres de gluten," Alimentos Hoy, vol. 22, no. 29, pp. 33-46, Ago. 2013.

[2] O. García, C. Aiello, M. Peña, J. Ruiz \& Y. I. Acevedo, "Caracterización físico-química y propiedades funcionales de la harina obtenida de granos de quinchoncho (Cajanus cajan (L.) Millsp.) sometidos a diferentes procesamientos" UDO Agríc., vol. 12, no. 4, pp. 919-928, Dec. 2012.

[3] E. Miquilena, A. Higuera y B. Rodríguez, "Evaluación de propiedades Funcionales de Cuatro Harinas de Semillas de Leguminosas Comestibles Cultivadas en Venezuela," Rev. Fac. Agron. (LUZ), vol. 33, no. 1, pp. 58-75, Feb. 2016.

[4] Y. Marrugo-Ligardo, P. Montero-Castillo y M. Durán-Lengua, "Evaluación Nutricional de Concentrados Proteicos de Phaseolus lunatus y Vigna unguiculata," Inf. Tecnol., vol. 27, no. 6, pp. 107-114, Dic. 2016. https://doi.org/10.4067/ S0718-07642016000600011
[5] A. Chaparro, J. Gil e I. Aristizabal, "Efecto de la Hidratación y la Cocción en las Propiedades Físicas y Funcionales de la Harina de Vitabosa (Mucuna deeringiana)," Vitae, vol. 18, no. 2, pp. 133-143, Jul. 2011.

[6] J. López-Alcocer, R. Lépiz-Ildefonso, D. González-Eguiarte, R. Rodríguez-Macías y E. López-Alcocer, "Variabilidad morfológica de phaseolus lunatus 1 . silvestre de la región occidental de México," Rev. Fitotec. Mex., vol. 39, no. 1, pp. 49-58, Ene. 2016.

[7] C. Ruiz, C. Díaz, J. Anaya y R. Roja, "Análisis proximal, Antinutrientes, perfil de Ácidos Grasos y de Aminoácidos de Semillas y Tortas de 2 Especies de Sacha Inchi (Plukenetia Volubilis y Plukenetia Huayllabambana)," Rev. Soc. Quím. Perú., vol. 79, no. 1, pp. 29-79, Ene. 2013.

[8] M. V. Ruiz, "Efecto de dos procesos térmicos sobre la calidad biológica de la Proteína de grano de Guandul (Cajanus sp) y Vitabosa (Mucuna sp) utilizando como modelo animal pollos de engorde," tesis magistral, Depto. Prod. Anim., UNAL, Medellín, Colombia, 2015.

[9] M. Sibt-e-Abbas, M. S. Butt, M. T. Sultan, M. K. Sharif, A. N. Ahmad \& R. Batool, "Nutritional and functional properties of protein isolates extracted from defatted peanut flour," IFRJ, vol 22, no. 4, pp. 1533-1537, Jan. 2015.

[10] R. Vegas, A. Zabaleta y C. Vegas, "Efecto del pH Cloruro de sodio sobre las propiedades funcionales de harina de semilla de lupinus mutabilis "tarwi" variedad criolla," Agroind. sci., vol. 7, no. 1, pp. 49-55, Jun. 2018. https:// doi.org/10.17268/agroind.science.2017.01.05

[11] A. M. Hernández, S. P. Galván, G. Arrázola y J. Osorio, "Evaluación de las condiciones de proceso sobre las propiedades funcionales de harina de plátano", Rev. Agron. Col., vol. 34. no. 1 supl, pp. 560-562, Sept. 2016.

[12] S. Badui, Química de los alimentos, 4 ed., vol. 7, Naucalpan de Juárez, México: Pearson Educación, 2006.

[13] Y. E. García-Pacheco, M. Prieto y C. Fuenmayor, "Cinética, modelación y pérdidas de carotenoides para el secado de ahuyama (Cucurbita moschata) en cubos," Rev. Agron. Col., vol. 34. no. 1 supl, pp. 573-576, Sept. 2016.

[14] Official Methods of Analysis, 18th ed., Official Method 2005.08, AOAC International, AOAC, Gaithersburg, MD, USA, 2005.

[15] ISO 1871:2009(en), Food and feed products - General guidelines for the determination of nitrogen by the Kjeldahl method, International Organization For Standardization, ISO, Geneva, Switzerlan, 2009

[16] P. J. Espitia-Pérez, Y. J. Pardo-Plaza y A. P. MontalvoPuente, "Proximate analysis characteristics of flours obtained from Papocho and Pelipita plantains (Musa ABB Simmonds)," Acta Agron., vol. 62, no. 3, pp. 189-195, Dic. 2013.

[17] Y. Balbín, "Influencia de la Cocción por Vía Húmeda y Seca en las Propiedades Funcionales de Harina de Semilla de Calabaza," tesis pregrado, Fac. Ing. Ind. Alim., UNCP, Huancayo, Pe, 2018.

[18] A. Padilla, "Efecto de diferentes tipos de cocción sobre el contenido en humedad, lípidos, proteínas y sales minerales de la caballa (Scomber)," tesis grado, Fac. Bio., ULL, San Cristóbal de La Laguna, Tenerife, España, 2015

[19] E. I. Hernández, W. A. Blandón, R. Escorcia y S. L. Blandón, "Producción de Harina de Frijoles (Phaseolus Vulgaris) y Evaluación Sensorial," El Higo, vol. 7, no. 1, pp. 11-17, Dic. 2017. https://doi.org/10.5377/elhigo.v7i1.8616

[20] K. Carović-Stanko, M. Maloić, J. Pintar, Z. Liber, I. Radosavljević, D. Bedeković, S. Guberac, V. Očić \& B, Lazarević, "Nutritional quality of phaseolin types and morphotypes of green bean (Phaseolus Vulgaris L.)" Agrociencia, vol. 52, no. 4, pp. 523-537, Jan. 2018. 
[21] L. Serna-Cock, O. Pabón-Rodríguez y J. Quintana-Moren, "Efectos de la Fuerza Iónica y el Tiempo de Remojo de Legumbres Secas sobre sus Propiedades Tecnofuncionales," Inf. Tecnol., vol. 30, no. 2, pp. 201-210, Mar. 2019. https:// doi.org/10.4067/S0718-07642019000200201

[22] M. Sánchez-Arteaga, E. Urías-Silvas, H. Espinosa-Andrews \& E. García-Márquez, "Effect of chemical composition and thermal properties on the cooking quality of common beans (Phaseolus vulgaris)," CyTA, vol. 13, no. 3, pp. 497-502, Dec. 2014. https://doi.org/10.1080/194763 37.2014 .988182

[23] G. Barraza, J. Soriano y C. Quevedo, "Perspectivas tecnológicas y nutricionales de la fibra dietética," Pueblo cont., vol. 18, no. 2, pp. 253-265, Dec. 2007.

[24] J. Vásquez y M. Argüelles, "Características Físicas, Químicas y Funcionales de la Harina de Quinua Orgánica (Chenopodium Quinoa Willd)," Ciencia y tecnología de Alimentos, vol. 27, no. 1, pp. 7-11, 2017.

[25] C. Granados, L. E. Guzman, D. Acevedo, M. Díaz y A. Herrera, "Propiedades Funcionales del Almidón de Sagu (Maranta arundinacea)," Biotecnol. Sector Agropecuario Agroind., vol. 12, no. 2, pp. 90-96, Feb. 2014.

[26] P. Pineda-Gómez, D. F. Coral, M. L. Arciniegas, A. Rorales-Rivera y M. E. Rodríguez, "Papel del agua en la gelatinización del almidón de maíz: estudio por calorimetría diferencial de barrido," Ing. Cienc., vol. 6, no. 11, pp. 131-133, Mar. 2010.

[27] M. Granito, Y. Brito \& A. Torres, "Chemical composition, antioxidant capacity and functionality of raw and processed Phaseolus lunatus," J. Sci. Food Agr., vol. 87, no. 5, pp. 2801-2809, Sept. 2007. https://doi.org/10.1002/ jsfa. 2926

[28] Y. Aguilera, I. Estrella, V. Benitez, R. M. Esteban \& M. A. Martín-Cabrejasa, "Bioactive phenolic compounds and functional properties of dehydrated bean flours," Food Res. Int., vol. 44, no. 3, pp. 774-780, Apr. 2011. https://doi. org/10.1016/j.foodres.2011.01.004

[29] C. Enyinnaya, O. Adewuyia \& J. Oneh, "Effect of germination on the chemical, functional and pasting properties of flour from brown and yellow varieties of tigernut (Cyperus esculentus)," Food Res. Int., vol. 42, no. 8, pp. 1004-1009, Oct. 2009. https://doi.org/10.1016/j.foodres.2009.04.024

[30] D. Romero y L. Tuiran, "Caracterización fisicoquímica, funcional, reológica y composicional de la harina precocida de cubio (Tropaeolum tuberosum R\&P) cultivado en diferentes fuentes de fertilización," trabajo grado, Fac. Ing., Progr. Ing. Alim., ULSA, Bogotá, D.C., Colombia, 2017.

[31] J. Torres, K. González, D. Acevedo y J. Jaimes, "Efecto de la utilización de harina de Lens culinaris como extensor en las características físicas y aceptabilidad de una salchicha," Revista Tecnura, vol. 20, no 49, pp. 15-28, Jul. 2016.
[32] A. García y E. Pacheco-Delahaye, "Evaluación de una Bebida Láctea Instantánea a base de Harina de Arracacha (Arracacia Xanthorrhiza) con la adición de Ácido Fólico," Rev. Chil. Nutr., vol. 37, no. 4, pp. 480-492, Dic. 2014. https://doi.org/10.4067/S0717-75182010000400009

[33] C. L. Navarro, D. Restrepo y J. Pérez, "EL GUANDUL (Cajanus cajan) Una Alternativa en la Industria de los Alimentos," Biotecnol. Sector Agropecuario Agroind., vol. 12, no. 2, pp. 197-206, Jul. 2014.

Yair García Pacheco es Ingeniero Agroindustrial en 2005 de la Universidad del Atlántico (Barranquilla, Colombia) con maestría en Seguridad Alimentaria y Nutricional en 2017 de la Universidad del Atlántico (Barranquilla, Colombia)y experiencia en el área agroindustrial y seguridad alimentaria. Trabajos de Investigación en las áreas de agroindustria y seguridad alimentaria; algunos de ellos relacionados con el tema de cinéticas de secado, degradación de compuestos bioactivos en matrices alimentaria y diseño de alimentos funcionales. Investigador miembro del grupo de investigación agroindustrial GIA (COL0028059) del Programa de Ingeniería Agroindustrial de la Universidad del Atlántico. https://orcid.org/0000-0003-0974-5650

Danelys Cabrera Mercado es Ingeniera Industrial de la Universidad de la costa (Barranquilla, Colombia), Maestría en Ingeniería (Cursando), de la Universidad de la Costa (Barranquilla, Colombia), con experiencia en docencia universitaria en al área de innovación. Trabajos de Investigación en las áreas de visibilidad web, Cienciometría, y agroindustria; Investigador miembro del grupo de investigación Producom (COL0028059) del Programa de Ingeniería Industrial de la Universidad de la costa. https://orcid.org/00000002-9486-9764

Jairo Andrés Ballestas Santos es Ingeniero agroindustrial de la Universidad del Atlántico (Barranquilla, Colombia) con Diplomado en SG-SST y Curso en gestión de la calidad. https://orcid.org/0000-00019204-4905

Michel José Campo Arrieta es Ingeniero Agroindustrial en 2019 de la Universidad del Atlántico (Barranquilla- Colombia). https://orcid.org/0000-00031709-0121 